\title{
Patients' Opinions Regarding Research and Management of Abdominal Aortic Aneurysms
}

\author{
Regent Lee, Amy Jones, Ismail Cassimjee, Ashok Handa \\ Nuffield Department of Surgical Sciences, \\ University of Oxford, UK \\ Corresponding Author: \\ Regent Lee \\ regent.lee@nds.ox.ac.uk
}

Keywords: Abdominal Aortic Aneurysm, Patient Preferences, biomarkers, patient and public involvement 
2 The epidemiology of abdominal aortic aneurysm (AAA) is changing. Outcomes for aortic surgery have improved. Biomarkers of AAA progression are emerging. We recently reported the opinions of international vascular surgery colleagues regarding research and management for AAAs. This study aimed to ascertain a real-world patients' opinion regarding the same questions.

\section{Method}

We administered a survey to patients with AAAs. We first ascertained their views on the priority topics for research in AAA. Using contemporary epidemiologic and surgical outcome data. We asked their preferences for different aspects of management in the hypothetical scenario where they had been diagnosed with a small $(4 \mathrm{~cm}) \mathrm{AAA}$ and a hypothetical biomarker predicted it to be fast growing.

\section{Results}

12 We received 191 responses from patient with AAAs (males 91\%, median-age-group 75-79 years). Amongst the topics of research for AAA, the top priorities for research chosen by patients were: "discovering why AAA develops in a person" and "discovering new medications that can make an AAA shrink back to normal size". In the hypothetical scenario, $42 \%$ of patients would prefer to have surgery early, while they are younger and fitter. $52 \%$ would follow the surgeon's recommendation as to

17 whether to have early surgery or not. A high proportion of respondents would likely consider taking part in a clinical trial to test if early surgery in such a scenario will be beneficial.

\section{Conclusion}

The results represent a snapshot of patient's views in terms of priorities for AAA research. We further demonstrate how patient's opinions can signpost the potential path for biomarker research to impact clinical practice. 
24 Outcomes for aortic surgery have improved significantly over time. The risk of 30-day surgical mortality from open surgical repair (OSR) of AAAs was historically bench marked at $\sim 8 \%$, whereby it crossed the annual risk of rupture of AAAs with a size of $5.5 \mathrm{~cm} .{ }^{1,2}$ Landmark trials of surgery for small AAAs, which included patients with AAA sizes between $4.0-5.5 \mathrm{~cm}$ and the subgroup with previous rapid expansion (5-10mm/year) have failed to demonstrate overall survival benefits of surgery (OSR or EVAR) in these patients. ${ }^{3}$ However, these trials did not employ other strategies for stratifying patients who are at risk of rapid future AAA progression.

The epidemiology of AAA is also changing. Patients are being diagnosed with AAAs at an increasingly older age ${ }^{4}$; Observations from large AAA cohorts show that patients with AAAs are at higher risk of developing incident cardiovascular morbidities and mortality as compared to their contemporaries. ${ }^{5}$

${ }^{6}$ Under the current practice of surveillance for small AAA, patients are being diagnosed at an older age, and are yet subject to the same surveillance strategy. During this period, patients may have developed new medical co-morbidities and render them physically unfit for surgery despite reasonable life expectancy. Given the pace of modern day scientific discovery, novel biomarker(s) for AAA progression may be emerging ${ }^{7,8}$. Such biomarker(s) may allow the identification of the group of patients who may benefit from early AAA surgery.

We recently conducted an international survey of vascular surgery professionals regarding their opinion on the priorities for AAA research, and showcased the potential path for biomarker research to impact clinical management ${ }^{9}$. There is increasing emphasis on patient views regarding disease treatment and clinical research both for health care delivery and resource allocations ${ }^{10}$, yet there is no published literature regarding patient preferences for treatment options for small AAAs in the contemporaneous settings.

The aims of our study are therefore to ascertain the a real-world patients' views on what is important for AAA research, and their preferences for the management of small AAAs in the settings where a 
precision care model is available to stratify their care based the AAA growth rate rather than the AAA size.

Methods

We delivered a survey questionnaire to every patient on the registry of the Oxford Regional Vascular Services, Unite Kingdom. (Oxford Abdominal Aortic Aneurysm study, UK Southwest Research Ethics Committee approval Ref: 13/SW/250). The survey was also made available through several internet sites in order to allow participants to respond directly online. (http://tinyurl.com/oxaaasurvey2016) The survey was first drafted by the research team, with further refinements by a focus group which consisted of AAA patients and members of the public ${ }^{11}$.

In this survey, participants were given the option to remain anonymous but asked provided basic demographic details such as their age group and gender. As part of the survey, we questioned what they viewed as important in terms of AAA research by providing a list of research questions (listed in Figure 1), asking them to choose the five that they viewed as more important areas for research.

In the subsequent section of the survey, we presented the participants with a hypothetical scenario to ascertain their preference in terms of treatment for small AAAs. In the scenario, the participant had just been diagnosed with a small AAA $(4 \mathrm{~cm})$. A brief outline highlighted the epidemiologic data of patients with AAAs, including the increased chance of developing other cardiovascular co-morbidities while under surveillance as compared to those without AAAs. We quoted contemporary surgical outcome data [ $<2 \%$ mortality for routine open surgical repair (OSR), $<0.5 \%$ mortality for endovascular repair (EVAR)], routine recovery time and long term follow up plans for surgery. In addition, we introduced a hypothetical novel biomarker which predicted their AAA to be faster growing, and would likely to reach existing surgical threshold within the next 3-5 years. The participants were told to assume that they were fit to undergo either OSR or EVAR at this moment in time. 
Using this scenario, we ascertained their preferences regarding the following: (a) whether they would prefer to have surgery early or later; (b) what type of surgery would they prefer if they chose to have surgery early; (c) would they be willing to take part in a trial to test whether early surgery will be beneficial in such settings; (d) what they view as most important to them in a trial to test whether early surgery would be beneficial in such settings.

\section{Results}

From March 2016, we sent out some 600 surveys through the post. The online survey were also listed on the following sites to allow invited participants to directly enter their : (a) OxAAA webpage: Nuffield Department of Surgical Sciences, University of Oxford. (http://www.nds.ox.ac.uk/research/oxaaa) (b) Patients Active in Research Thames Valley (http://patientsactiveinresearch.org.uk/oxaaa-survey-tellus-what-you-think-is-important).

We received 191 responses from patient with AAAs. Ninety-six percent of these responses were reived within the first three months. Males represented $91 \%$ of the respondents. The median age group of all respondents was $75-79$ years, with $38 \%$ had already undergone AAA surgery. The frequency of choice for each of the nine research questions is summarised in Figure 1, with the most frequently chosen being: "discovering why an AAA develops in a person" (83\%), "discovering new medications that can make AAA shrink back to normal size" (71\%), "discovering new medications that can stop an AAA from growing further" (64\%).

In the hypothetical scenario where the biomarker predicts the AAA will be fast growing and therefore likely to reach operative threshold in a few years, $42 \%$ of respondents would prefer to have surgery early, while they were comparatively younger and fit. Very few preferred deferring surgery (2\%) or were unsure (4\%). The majority (52\%) of respondents would follow the surgeon's recommendation (SR) as to whether to have early surgery or not. Amongst those who would prefer to have earlier surgery, $27 \%$ preferred OSR, $30 \%$ preferred EVAR, while $43 \%$ would follow the surgeon's recommendation (SR) in terms of the type of surgical repair to undertake. (Figure 2 - top panel). 
A high proportion of respondents would consider taking part in a clinical trial to test if early surgery in such a scenario will be beneficial in such a setting. (yes definitely: $20 \%$; maybe: $21 \%$; $51 \%$ would follow SR). When asked what they viewed as more important in terms of benefit from having surgery earlier, many chose improvements in quality of life (35\%), or reductions in their anxiety about having an AAA (27\%) as more important than making them live longer (38\%) (Figure 2 - lower panel). There was no difference in the response given by patients who have already had surgery for their AAAs, as compared to those who have not had surgery (data not shown).

When the responses were analysed in subgroups according to the participant age groups by median split (group 1: $<75$ years old, $n=76$; group 2: $\geq 75$ years old, $n=115$ ), There were no differences observed in terms of their preferences on timing of surgery for small AAAs, the type of surgery if they preferred to have surgery early, or what they view as important in terms of the outcome measure for a trial (data not shown).

\section{Discussion}

This is the first time a study highlights the "real-world" patients' views on the priorities for AAA research, and their preferences in terms of timing for surgery of small AAAs taking into consideration other contemporary factors including the hypothetic advent of a biomarker of AAA progression. With the increasing need for researchers to demonstrate the need for specific research, and a projected path for their research to impact clinical practice, these are important findings to researchers in the subject of AAA disease.

The management of abdominal aortic aneurysm is well defined in literature, with a recognised threshold for surgery at $5.5 \mathrm{~cm}$. Trials on small AAAs have shown no benefit of early surgery compared to a surveillance strategy up to the intermediate term, as early surgery confers the immediate operative risk not incurred by the surveillance strategy ${ }^{3}$. As such current NHS, AHA, and ESC guidelines do not recommend surgery until an AAA reaches $5.5 \mathrm{~cm}^{12-14}$. 
However, these landmark trials on surgery for small AAAs have also shown that the rates of AAA progression vary greatly between individuals, and that the majority with moderate size AAAs ( 70\%) will progress to require surgery during the next 5 years $^{15,16}$. It is a reasonable hypothesis that biomarkers for future AAA progression can have impact on patient management. The notion that patients with AAAs that will be growing faster may benefit from surgery early requires testing by randomised trials, or justification by comprehensive health economics analysis that takes into account of the patients' values.

More importantly, this notion further relies on the assumption that patients would actually want to have surgery early. It is reasonable to argue that patients may not wish to have surgery early before reaching the recognised $5.5 \mathrm{~cm}$ threshold, which would render the arguments for early surgery irrelevant. Our data provides direct evidence that patients are inclined to have early surgery when told that their AAAs will be fast growing. In our recent international survey to vascular surgery colleagues, we asked our colleagues to what they would do in this situation, if they were the patient themselves. The majority of vascular surgery colleagues prefer to have early surgery rather than surveillance in such a scenario ${ }^{9}$. This is a strong supporting evidence for the notion above.

Another evidence to hint at patients' willingness to undertake surgery early lies in the great regional variations between the adherence to surgical threshold for AAA repair. In the United states, some $40 \%$ of patients receive AAA surgery below $5.5 \mathrm{~cm}^{17}$, while this number is much lower for the $U K^{18,19}$. Notwithstanding the differences in the health care systems, patient preferences must have clear influence in the decision making process for surgery in countries with low adherence for the regarded $5.5 \mathrm{~cm}$ threshold.

In this survey, we set the hypothetical scenario of the patient having a small AAA at $4 \mathrm{~cm}$ because all the existing major trials comparing early surgery vs surveillance for small AAAs, whether by OSR or EVAR, have used this as the threshold as an inclusion criteria (UKSAT, ADAM, CAESAR, PIVOTAL). Although some of smaller AAAs (between 3 to $4 \mathrm{~cm}$ ) may also be fast growing in the coming year(s), 
we reasoned surgeons would be unlikely to offer surgery when the AAAs smaller than $4 \mathrm{~cm}$ even in the setting of a trial. The $4 \mathrm{~cm}$ threshold in this survey was therefore guided by existing literature and pragmatism.

Our study provides specific patient and public involvement data that is relevant to future translational research of AAA biomarkers. In modern day medical practice, increasing emphasis is placed on patient values and preferences, with a paradigm shift towards a model of shared decision making for treatment options between patients and physicians. Similarly, patient and public involvement (PPI) is becoming an integral part of contemporary biomedical research, recognising the importance of patient input into every stage of research. ${ }^{10}$ Nevertheless, we appreciate many patients still entrust the decision making process based on the Physician's recommendations, evident by the high proportion of respondents who would make their decisions based on surgeon's recommendations (on the timing of surgery and the type of surgery).

Our survey findings are based on the hypothetical scenario in the advent of biomarker(s) for the prediction of future AAA growth. This is irrespective of the type of biomarker(s) that may become available. Although there is as yet no biomarker validated for this purpose, with the plethora of ongoing biomedical research it will be a matter of time before a holy grail is found. The ascertainment of patient's preferences for early surgery in such settings, as reported by our findings, is a fundamental requisite for the future research required to validate such biomarker(s).

\section{Conclusion}

Our survey results represent a snapshot of patient's views in terms of priorities for AAA research. We further demonstrate how patient's opinions can signpost the potential path for research to impact clinical practice. 
170 We acknowledge the support by the following: Patient Active in Research Thames Valley; Oxford 171 Biomedical Research Centre; Research Development Services Southcentral Region UK; Medical 172 Research Fund by Medical Sciences Division. National Institute of Health Research Oxford Biomedical 173 Research Centre. University of Oxford Vice Chancellor's Public Engagement in Research Seed Award. 174 We further acknowledge the input of the following to the OxAAA Survey: Lucy Fulford-Smith, Felicity 175 Woodgate, Kirthi Bellamkonda, Katherine Hurst, Matthew Williams, James Taylor.

176 
179 1. Programme VSQI. 2015 NVR Annual Report | VSQIP. 2016.

180 2. Brewster DC, Cronenwett JL, Hallett JW, Jr., Johnston KW, Krupski WC and Matsumura JS. Guidelines for the treatment of abdominal aortic aneurysms. Report of a subcommittee of the Joint Council of the American Association for Vascular Surgery and Society for Vascular Surgery. J Vasc Surg. 2003;37:1106-17.

3. Filardo G, Powell JT, Martinez MA and Ballard DJ. Surgery for small asymptomatic abdominal aortic aneurysms. Cochrane Database Syst Rev. 2015:Cd001835.

4. Choke E, Vijaynagar B, Thompson J, Nasim A, Bown MJ and Sayers RD. Changing epidemiology of abdominal aortic aneurysms in England and Wales: older and more benign? Circulation. 2012;125:1617-25.

5. Norman $\mathrm{P}$, Le $\mathrm{M}$, Pearce $\mathrm{C}$ and Jamrozik K. Infrarenal aortic diameter predicts all-cause mortality. Arterioscler Thromb Vasc Biol. 2004;24:1278-82.

6. Duncan JL, Harrild KA, Iversen L, Lee AJ and Godden DJ. Long term outcomes in men screened for abdominal aortic aneurysm: prospective cohort study. Bmj. 2012;344:e2958.

7. Wanhainen A, Mani K, Vorkapic E, De Basso R, Bjorck M, Lanne T and Wagsater D. Screening of circulating microRNA biomarkers for prevalence of abdominal aortic aneurysm and aneurysm growth. Atherosclerosis. 2017;256:82-88.

8. Lee R, Bellamkonda K, Jones A, Killough N, Woodgate F, Williams M, Cassimjee I and Handa A. Flow Mediated Dilatation and Progression of Abdominal Aortic Aneurysms. Eur J Vasc Endovasc Surg. 2017.

9. Lee R, Jones A, Cassimjee I and Handa A. International Opinion on Priorities for Research for Small Abdominal Aortic Aneurysms and the Potential Path For Research to Impact Clinical Management. International Journal of Cardiology. 2017.

10. Petit-Zeman S and Locock L. Health care: Bring on the evidence. Nature News. 2013;501:160. 11. Lee R, Jones A, Woodgate F, Bellamkonda K, Killough N, Fulford-Smith L, Hurst K, Cassimjee I and Handa A. The Experience of Patients During the Clinical Management Pathway of Abdominal Aortic Aneurysms at a NHS Trust. http://dxdoiorg/101177/2374373517715010. 2017.

12. NHS. Abdominal aortic aneurysm - Treatment - NHS Choices. 2016.

13. Anderson JL, Halperin JL, Albert NM, Bozkurt B, Brindis RG, Curtis LH, DeMets D, Guyton RA, Hochman JS, Kovacs RJ, Ohman EM, Pressler SJ, Sellke FW and Shen WK. Management of patients with peripheral artery disease (compilation of 2005 and 2011 ACCF/AHA guideline recommendations): a report of the American College of Cardiology Foundation/American Heart Association Task Force on Practice Guidelines. Circulation. 2013;127:1425-43.

14. Erbel R, Aboyans V, Boileau C, Bossone E, Bartolomeo RD, Eggebrecht H, Evangelista A, Falk V, Frank H, Gaemperli O, Grabenwoger M, Haverich A, lung B, Manolis AJ, Meijboom F, Nienaber CA, Roffi M, Rousseau H, Sechtem U, Sirnes PA, Allmen RS and Vrints CJ. 2014 ESC Guidelines on the diagnosis and treatment of aortic diseases: Document covering acute and chronic aortic diseases of the thoracic and abdominal aorta of the adult. The Task Force for the Diagnosis and Treatment of Aortic Diseases of the European Society of Cardiology (ESC). Eur Heart J. 2014;35:2873-926.

15. Lederle FA, Wilson SE, Johnson GR, Reinke DB, Littooy FN, Acher CW, Ballard DJ, Messina LM, Gordon IL, Chute EP, Krupski WC, Busuttil SJ, Barone GW, Sparks S, Graham LM, Rapp JH, Makaroun MS, Moneta GL, Cambria RA, Makhoul RG, Eton D, Ansel HJ, Freischlag JA and Bandyk D. Immediate repair compared with surveillance of small abdominal aortic aneurysms. N Engl J Med. 2002;346:143744.

16. Mortality results for randomised controlled trial of early elective surgery or ultrasonographic surveillance for small abdominal aortic aneurysms. The UK Small Aneurysm Trial Participants. Lancet. 1998;352:1649-55.

17. Benarroch-Gampel J. SCVS - Does Aneurysm Size Matter?: Lack of Adherence to Diameter Threshold Criteria for Repair of Abdominal Aortic Aneurysms. 2016. 
18. Beck AW, Sedrakyan A, Mao J, Venermo M, Faizer R, Debus S, Behrendt C-A, Scali ST, Altreuther M, Schermerhorn M, Beiles B, Szeberin Z, Eldrup N, Danielsson G, Thomson I, Wigger PF, Björck M, Cronenwett JL, Mani $\mathrm{K}$ and Registries obotlCoV. Variations in Abdominal Aortic Aneurysm Care: A Report from the International Consortium of Vascular Registries. 2016.

232 19. Karthikesalingam A, Vidal-Diez A, Holt PJ, Loftus IM, Schermerhorn ML, Soden PA, Landon BE and Thompson MM. Thresholds for Abdominal Aortic Aneurysm Repair in England and the United States. http://dxdoiorg/101056/NEJMoa1600931. 2016. 
Figure 1: The assignment of importance on individual research questions. In this survey, patients were allowed to choose up to 5 research questions that they viewed as important for research. We received responses from 191 patients. This figure shows the frequency distribution their selections.

Figure 2: Patients' preference regarding the timing of surgery and the preferred type of surgery for managing small AAAs that will be fast progressing (Top). Willingness of the patients to take part in a trial to assess the benefit of early surgery for small AAAs that will be fast progressing, and the assignment of importance for outcome measures (bottom). In this survey, participants were posted with a hypothetical scenario where a biomarker predicts the small AAA $(4 \mathrm{~cm})$ will be fast growing and therefore likely to reach operative threshold in a few years. The top left panel shows their preferences in terms of their preference for the timing for surgery. The top right panel shows their preferences in terms of the type of surgical repair they prefer to undertake, assuming they are fit for either type of surgery. For those who preferred to have surgery early in the hypothetical scenario where a biomarker predicts the small AAA $(4 \mathrm{~cm})$ will be fast growing $(n=81)$, we asked whether they would be willing to take part in a clinical trial to test if early surgery would be beneficial in such a setting (bottom left panel). For all respondents ( $n=191)$, we asked what was most important to them in terms of the primary outcome measure (bottom right panel). Open surgical repair (OSR); endovascular repair (EVAR), follow the surgeon's recommendation (SR.) 